\title{
A Simple Method for Molecular Detection of Swine-Origin and Human-Origin Influenza A Virus
}

\author{
Laetitia Ninove, ${ }^{1,2}$ Céline Gazin, ${ }^{2}$ Ernest A. Gould, ${ }^{1,3}$ Antoine Nougairede, ${ }^{1,2}$ Antoine Flahault, ${ }^{4}$ \\ Rémi N. Charrel, ${ }^{1,2}$ Christine Zandotti, ${ }^{1,2}$ and Xavier de Lamballerie ${ }^{1,2,4}$
}

\begin{abstract}
Here we present a real-time one-step reverse transcriptase-polymerase chain reaction SYBR Green assay derived from the method reported by van Elden and collaborators (2001) that ensures the rapid, sensitive, and costeffective detection of both seasonal influenza A virus and emerging (H1N1) swine-origin influenza A virus (S-OIV). In addition to this screening test, which successfully detected both seasonal influenza A virus and S-OIV in human clinical samples, we showed that the probe initially designed by van Elden and collaborators could detect seasonal influenza A virus, but not S-OIV; a new probe was designed and tested that specifically detects $\mathrm{S}-\mathrm{OIV}$, but not seasonal influenza A. Both probe-based assays were validated by testing human clinical samples and specifically detected either seasonal influenza virus or S-OIV. Finally, in silico analysis of databases predicted that minor modifications of the van Elden primers would facilitate the use of this assay for the broad spectrum detection of all currently characterized variants of influenza A virus, including avian strains.
\end{abstract}

Key Words: Arbovirus(es)—Chikungunya—Dengue virus—Tick-borne encephalitis—Vector-borne.

$\mathbf{M}$ OLECULAR DETECTION OF INFLUENZA A VIRUSES, in the form of real-time reverse transcriptase-polymerase chain reaction (RT-PCR), has been broadly adopted in medical diagnostic laboratories (Brown 2006), and thus routine diagnosis now includes these molecular techniques. However, the detection methods are directed at classical seasonal influenza viruses, currently, human H3N2 and H1N1, but not at the detection of avian or porcine viruses, which are rarely encountered in human medicine. Consequently, a large number of specific tests have recently been proposed for the detection of $\mathrm{H} 5 \mathrm{~N} 1$ avian viruses, after the significant emergence of human cases. However, assays designed to detect a large range of viral subtypes are rarely used for routine diagnosis. Nevertheless, such assays do exist and provided that they are precisely evaluated, merit more frequent use. Daum and collaborators (2007) proposed a panel of highly sensitive typing and subtyping protocols in a real-time RT-PCR format, and suggested that these tests offer significant utility in the context of a pandemic influenza outbreak. Other assays have been proposed (Whiley and Sloots 2005, Valle et al. 2006, Karlsson et al. 2007, Li et al. 2008), including commercial kits (Gharabaghi et al. 2008) and tests derived from subtyping assays taking advantage of conserved nucleotide regions in the hemagglutinin gene (Gall et al. 2008, Yacoub et al. 2009).

Since 2002, we have been using a real-time PCR assay designed by van Elden and collaborators (2001) for the routine molecular detection of influenza A virus. In this test, a $189 \mathrm{nt}$ fragment within the Matrix gene of the virus is amplified using the INFA-1 and INFA-23 primers and hybridized with the INFA-specific probe (see Fig. 1) using a two-step RT-PCR protocol (i.e., a reverse transcription reaction using random hexaprimers, followed by a real-time PCR reaction). This assay has proved to be robust and able to detect human influenza virus subtypes $\mathrm{H} 1 \mathrm{~N} 1$ and $\mathrm{H} 3 \mathrm{~N} 2$ in clinical samples. Moreover, it can be combined with the simultaneous detection of influenza B virus in a multiplex assay (van Elden et al. 2001).

The recent spread of the H1N1 swine-origin influenza A virus (S-OIV) led us to reconsider this assay. The Matrix gene of S-OIV is of porcine origin, and genetic analysis of sequences available in GenBank revealed an $\sim 13 \%$ nt divergence

\footnotetext{
${ }^{1}$ Unité des Virus Emergents, Faculté de Médecine de Marseille, Université de la Méditerranée \& Institut de Recherche pour le Développement, Marseille, France.

${ }^{2}$ Fédération de Microbiologie, Assistance Publique-Hôpitaux de Marseille, Marseille, France.

${ }^{3} \mathrm{CEH}$ Oxford, Oxford, United Kingdom.

${ }^{4}$ EHESP School of Public Health, Rennes, France.
} 
A

Influenza A/Canada-ON/RV1526/2009 (H1N1) (S-OIV) S-OIV synthetic control

(1977(H1)

Human Influenza A synthetic control

Influenza A/Canada-ON/RV1526/2009(H1N1) (S-OIV)

S-OIV Synthetic control

Influenza A/USSR/90/1977(H1N1)

Human Influenza A synthetic control
GGACTGCAGCGTAGACGCTTTGTCCAAAATGCCCTAAATGGGAATGGGGACCCGAACAACATGGATAGAGCAGTTAAACTATACAAGAAGCTCAAAAGAGAAATA

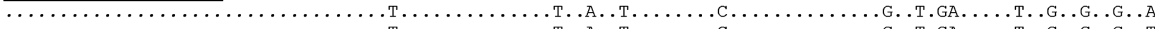
(1) ACGTTCCATGGGGCCAAGGAGGTGTCA CTAAGCTATTCAACTGGTGCACTTGCCA GTTGCATGGGCCTCATATACAACAGGATG

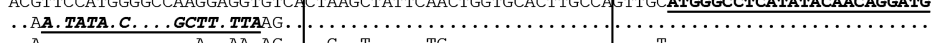

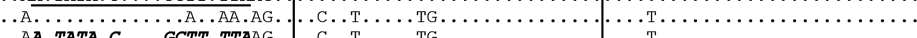

B

\begin{tabular}{|c|c|c|c|}
\hline Name & function & sequence & target \\
\hline $\begin{array}{l}\text { INFA-23 } \\
\text { panINFA-23 }\end{array}$ & $\begin{array}{l}\text { Sense primer } \\
\text { Reverse primer }\end{array}$ & $\begin{array}{l}\text { CAYTCTGTTGTATATGAGGCCCAT } \\
\text { CAYTCTGTTGTATATGAGNCCCAT }\end{array}$ & $\begin{array}{l}\text { Matrix gene positions } 405-382 \\
\text { Matrix gene positions } 405-382\end{array}$ \\
\hline S-INFA & probe & $\begin{array}{l}\text { FAM-CTCAGTTATTCTGCTGGTGCACTTGCCA-TAMRA } \\
\text { FAM-CTA_AGCTATTCAACTGGTGCACTTGCCA-TAMRA }\end{array}$ & $\begin{array}{c}\text { Matrix gene positions } 349-376 \\
\text { (seasonal Influenza A virus) } \\
\text { Matrix gene positions } 349-376 \\
\text { (Swine-origin H1N1 Influenza A virus) }\end{array}$ \\
\hline
\end{tabular}

FIG. 1. (A) Comparison of sequences of an S-OIV strain and a seasonal H1N1 strain in the region amplified by the Van Elden primers within the Matrix gene (positions 217-405). Dots denote identical sequence. Primers are bold and underlined; characters and the central box correspond to the sequence targeted by INFA and S-INFA probes. The sequence of synthetic RNA controls is also indicated. The $5^{\prime}$ end of these controls (first line) is identical and comes from the sequence of a human H1N1 strain (USSR/90/1977); the $3^{\prime}$ end (second line) is specific for human virus or S-OIV and includes a NotI sequence that is targeted by the NotI-P probe (in italics and underlined). (B) Primers and probes used in this study.

between the region targeted by the assay in the new porcine H1N1 virus and in previously circulating H1N1 or H3N2 human strains. In particular, the region chosen for probe hybridization includes four nt mismatches (see Fig. 1), suggesting that the probe initially designed by van Elden and collaborators would not efficiently detect the new porcine virus. In contrast, the van Elden primers matched the corresponding sequence of the new virus (Figs. 1 and 2).

Accordingly, the primers from the van Elden assay were used to elaborate a simple SYBR Green one-step real-time RT-PCR assay as described hereafter. Extraction: $200 \mu \mathrm{L}$ of nasopharyngeal sample was spiked with $5 \mu \mathrm{L}$ of MS2 phage suspension and used for nucleic acid extraction (elution volume: $90 \mu \mathrm{L}$ ) using the EZ1 Biorobot and the virus mini kit (both from Qiagen, Courtaboeuf, France). Amplification: one-step RT-PCR gene amplification was performed in a $25 \mu \mathrm{L}$ final volume, using $5 \mu \mathrm{L}$ of RNA extract, $1.4 \mu \mathrm{L}$ of each $10 \mu \mathrm{M}$ primer solution, and the one-step QuantiTect SYBR Green RT-PCR Kit master mix with Uracil-N-Glycosylase (UNG) (Qiagen).

Two distinct reactions were conducted using the Mx3005 ${ }^{\circledR}$ QPCR System thermocycler (Stratagene-Agilent, Massy, France) and the following thermal profile. Segment $1: 50^{\circ} \mathrm{C}$, 2 min; segment 2: $95^{\circ} \mathrm{C}, 15 \mathrm{~min}$; segment $3: 94^{\circ} \mathrm{C}, 15 \mathrm{~s} ; 60^{\circ} \mathrm{C}, 30 \mathrm{~s}$; and $72^{\circ} \mathrm{C}, 30 \mathrm{~s}(45 \times)$, followed by the analysis of a thermal dissociation curve. One reaction included the INFA-1/INFA-23 primer set for the detection of the influenza A virus; the other included the MS2-S2/-R2 primer set for detection of MS2 phage detection (see Fig. 1B for a list of primers and probes used in the current study). The latter reaction was used as an internal control for monitoring the extraction and RT-PCR steps, and in particular the detection of amplification inhibitors in the biological material tested (see the dissociation curve in Fig. 3, with a Tm at $82^{\circ} \mathrm{C}$ ).
In addition, synthetic RNA transcripts (including a specific extrinsic specific sequence, that is, a NotI restriction site; see Fig. 1A) were produced by amplification with a primer containing the T7 promoter sequence and by transcription with the T7-MEGA shortscript (Ambion, Applied Biosystems, Courtaboef, France) according to the manufacturer's instructions. They were quantified by spectrophotometry and used as positive controls for RT-PCR amplification.

Synthetic RNA controls were detected with a Tm of the amplicons at $79.5^{\circ} \mathrm{C}$ (see Fig. 3). When serial dilutions were tested, 50 RNA copies per reaction could be reproducibly and unambiguously detected (equivalent to $\sim 4.5$ viral RNA copies per $\mu \mathrm{L}$ of clinical sample tested; data not shown). With regard to these performances, the assay was subsequently used to detect genomic viral RNA in clinical samples. It was able to detect either classicalinfluenza A virus (see Fig. 3, detection of $\mathrm{H} 3 \mathrm{~N} 2$ virus in a human naso-pharyngeal sample, with a Tm at $80^{\circ} \mathrm{C}$ ) or the newly emerging $\mathrm{H} 1 \mathrm{~N} 1$ porcine virus (detection of S-OIV in a human naso-pharyngeal sample, with a Tm at $81^{\circ} \mathrm{C}$ ), justifying its use for the immediate detection of the influenza A virus in clinical samples.

In addition to this test, one-step real-time RT-PCR assays were performed using either the probe designed by van Elden and collaborators, or the equivalent probe (S-INFA) designed from the sequences available for S-OIV (see Fig. 1) and the following protocol. Amplification: Genetic amplification was performed in a one-step RT-PCR format and a $25 \mu \mathrm{L}$ final volume, using $5 \mu \mathrm{L}$ of RNA extract, $1 \mu \mathrm{L}$ of each $10 \mu \mathrm{M}$ primer solution, $0.4 \mu \mathrm{L}$ of $10 \mu \mathrm{M}$ probe solution, and the Super Script III Platinum One step qRT PCR System (Invitrogen, Cergy Pontoise, France). Two reactions were conducted using the Mx3005P QPCR System thermocycler and the following thermal profile. Segment 1: $48^{\circ} \mathrm{C}, 30 \mathrm{~min}$; segment 2: $95^{\circ} \mathrm{C}$, 
A

Forward primer INFA-1 (panINFA-1)

Avian strains, $\mathrm{N}=311$

Human strains, $\mathrm{N}=2461$

Swer strains,, $\mathrm{N}=344$

S-OIV strains, $N=57$

Reverse primer* INFA-23 (panINFA-23) Avian strains, $\mathrm{N}=3111$ Human strains, $\mathrm{N}=2461$

Swine strains**, $N=34$

Other strains, $N=168$

\begin{tabular}{cccccccccccccc|ccccccc|}
$\longrightarrow$ G & G & A & C & T & G & C & A & G & C & G & T & A & G & A & C & G & C & (N) & T & T \\
1 & 0 & 3 & 2 & 1 & 31 & 0 & 0 & 36 & 1 & 0 & 2 & 0 & 9 & 3 & 7 & 5 & 684 & $(0)$ & 0 & 0 \\
0 & 0 & 0 & 0 & 0 & 0 & 0 & 0 & 3 & 0 & 0 & 0 & 0 & 0 & 0 & 0 & 0 & 4 & $(0)$ & 0 & 0 \\
0 & 0 & 0 & 0 & 0 & 0 & 0 & 0 & 0 & 0 & 0 & 0 & 0 & 0 & 0 & 0 & 0 & 21 & $(0)$ & 0 & 0 \\
0 & 0 & 0 & 0 & 0 & 0 & 0 & 0 & 4 & 0 & 0 & 0 & 0 & 0 & 0 & 0 & 0 & 4 & $(0)$ & 0 & 0 \\
0 & 0 & 0 & 0 & 0 & 0 & 0 & 0 & 3 & 0 & 0 & 0 & 0 & 0 & 0 & 0 & 0 & 0 & $(0)$ & 0 & 0 \\
\hline
\end{tabular}

B

Avian strains, $\mathrm{N}=311$ Human strains, $\mathrm{N}=246$ Swine strains, $\mathrm{N}=344$ Other strains, $\mathrm{N}=168$

FIG. 2. (A) Number of nt mismatches observed between the primers used and sequences deposited in the Influenza Resource database for influenza A viruses of different origins. The sequence corresponding to the reverse primers is presented in the polarity of genomic sequence deposited in databases (reverse complement of the primer used for genetic amplification). ${ }^{* *}$ Swine strains do not include S-OIV strains, which are presented separately. The six terminal $3^{\prime}$ positions of the primers (essential for a correct hybridization and initiation of polymerization) are in boxes. Nucleotides in parentheses correspond to modifications of sequences proposed for primers panINFA-1 and panINFA-23. Modifications were proposed only for variable positions among the six last $3^{\prime}$ terminal nucleotides of each primer. The number of mismatches observed after introduction of these modifications are also indicated in parentheses. (B) Number of strains with a significant number of mismatches observed in the complete primers or in the six terminal $3^{\prime}$ positions of the primers.

2 min; segment $3: 95^{\circ} \mathrm{C}, 15 \mathrm{~s} ; 60^{\circ} \mathrm{C}, 1 \mathrm{~min}(45 \times)$. One reaction included the INFA-1/INFA-23 primer set and the INFA probe for the detection of the seasonal influenza A virus; the other included the same primers and the S-INFA probe for the detection of S-OIV. Synthetic RNA transcripts specific for human seasonal influenza A virus and S-OIV were used as positive controls for RT-PCR amplification.

Using both synthetic RNA transcripts and human samples positive for either H3N2 or S-OIV, it could be shown (see Fig. 3) (i) that the van Elden and S-OIV probes could efficiently detect the H3N2 and S-OIV, respectively, but (ii) that the van Elden probe could not detect S-OIV, while the S-OIV probe could not detect seasonal influenza virus. A probe designed to detect specifically the synthetic positive control (NotI-P) was used to rule out the possibility of accidental amplification of the control.

Altogether, these results suggest that this SYBR Green onestep real-time RT-PCR assay is a simple, inexpensive, but very effective tool for screening clinical samples for the presence of
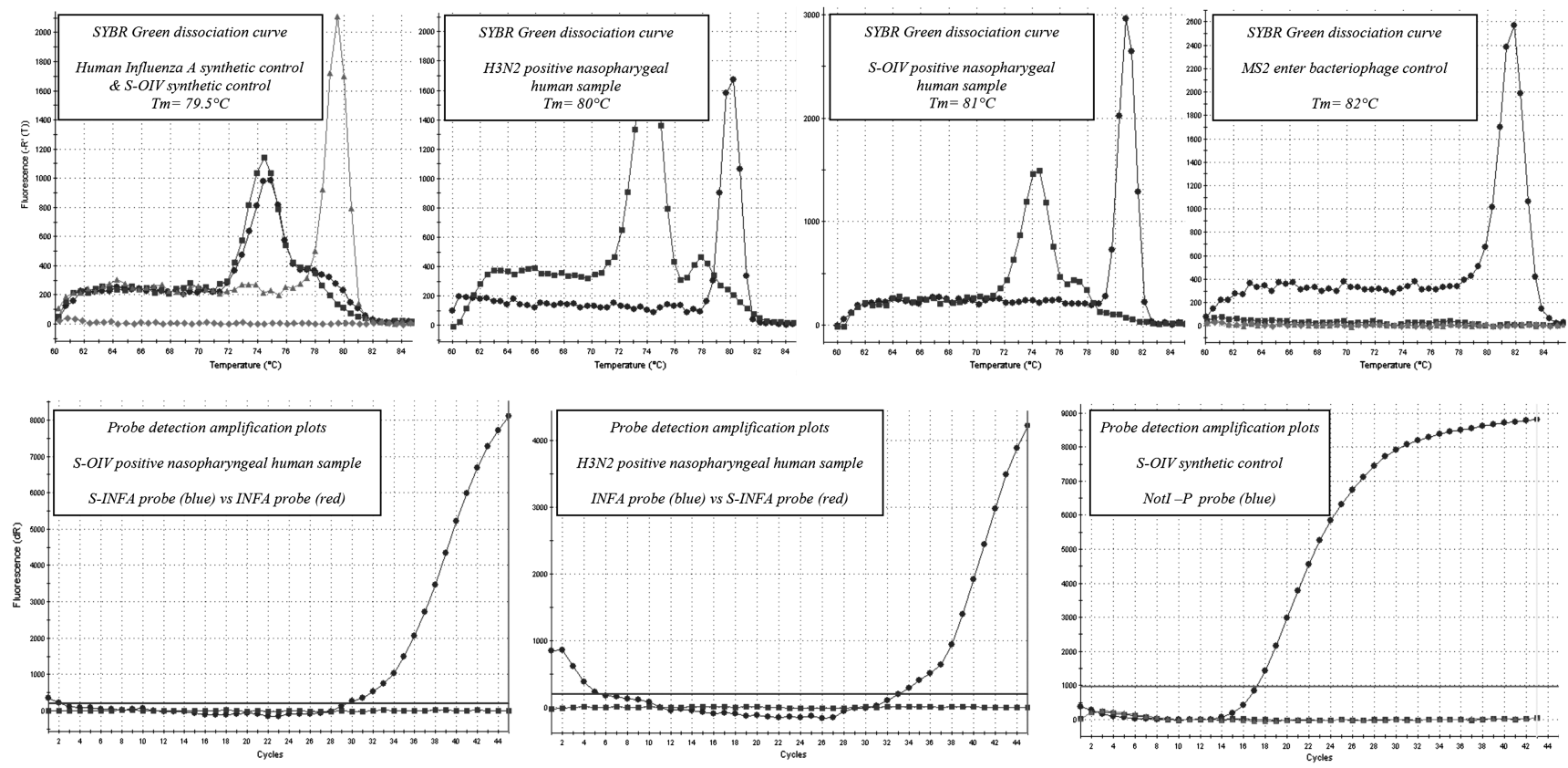

FIG. 3. Real-time reverse transcriptase-polymerase chain reaction amplification of synthetic controls and clinical samples. First line shows SYBR dissociation curves for influenza virus synthetic controls, H3N2-positive and S-OIV-positive clinical samples, and MS2 control. Second line shows specific probe detection of H3N2 and S-OIV in clinical samples using INFA and S-INFA probes that of S-OIV synthetic control using NotI-P probe (system designed for the detection of incidental contamination by synthetic controls). 
influenza A virus. Most importantly, positive samples may be secondarily tested using the same primers and the van Elden (INFA) and S-OIV (S-INFA) probes to discriminate between seasonal and S-OIV. It should also be noted that the direct sequencing of the products resulting from the SYBR Green assay produced the definitive differentiation of seasonal and S-OIV strains.

In addition to this initial study, which provides a rapid and pragmatic solution to the detection of the newly emerging S-OIV, we analyzed in depth:

(i) 6084 influenza A Matrix sequences available in the Influenza Virus Resource database (www.ncbi.nlm.nih .gov/genomes/flu/flu.html) for which hemagglutinin and neuraminidase subtyping was available. This data set included 3111 avian strains [H1N1 (73), H1N2 (5), H1N3 (4), H1N5 (2), H1N6 (4), H1N9 (1), H2N1 (14), H2N2 (8), H2N3 (25), H2N4 (1), H2N5 (10), H2N7 (7), H2N8 (4), H2N9 (14), H3N1 (8), H3N2 (57), H3N3 (5), H3N4 (3), H3N5 (7), H3N6 (44), H3N7 (3), H3N8 (96), H3N9 (2), H4N1 (3), H4N2 (15), H4N3 (5), H4N4 (2), H4N5 (2), H4N6 (91), H4N7 (1), H4N8 (35), H4N9 (8), H5N1 (980), H5N2 (126), H5N3 (50), H5N4 (4), H5N6 (1), H5N7 (6), H5N8 (4), H5N9 (5), H6N1 (145), H6N2 (92), H6N3 (4), H6N4 (6), H6N5 (19), H6N6 (9), H6N8 (47), H6N9 (2), H7N1 (43), H7N2 (121), H7N3 (91), H7N4 (4), H7N5 (2), H7N7 (29), H7N8 (4), H7N9 (4), H8N4 (18), H9N1 (6), H9N2 (524), H9N5 (4), H9N6 (2), H9N8 (2), H9N9 (1), H10N1 (2), H10N2 (2), H10N3 (3), H10N4 (3), H10N5 (6), H10N6 (2), H10N7 (37), H10N8 (6), H10N9 (2), H11N1 (8), H11N2 (9), H11N3 (7), H11N4 (1), H11N6 (5), H11N8 (2), H11N9 (42), H12N1 (1), H12N2 (1), H12N4 (3), H12N5 (23), H12N8 (1), H12N9 (2), H13N2 (5), H13N3 (1), H13N6 (11), H13N8 (1), H13N9 (3), H14N5 (1), H14N6 (1), H15N9 (1), H16N3 (5)], 2461 human strains [H1N1 (696), H1N2 (35), H2N2 (76), H3N2 (1532), H5N1 (119), H7N2 (1), H7N7 (2)], 344 porcine strains [H1N1 (158), H1N2 (56), H3N1 (2), H3N2 (92), H3N3 (2), H4N6 (2), H5N1 (5), H5N2 (1), H9N2 (26)], and 168 strains of diverse origin (environment, horse, tiger, leopard, cat, civet, musk rat, mink, etc.) [H11N6 (1), H11N8 (2), H11N9 (1), H1N1 (1), H3N2 (3), H3N6 (3), H3N8 (80), H4N6 (2), H5N1 (18), H5N2 (7), H7N2 (47), H7N3 (2), H7N7 (1)];

(ii) $57 \mathrm{~S}-\mathrm{OIV}$ influenza A Matrix sequences available in GenBank.

The performance of the van Elden primers was evaluated in silico for each group of influenza A viruses (see Fig. 2). This analysis revealed that these primers are indeed highly appropriate for the detection of human seasonal influenza A virus and for most porcine strains, including the newly emerging S-OIV. However, minor but important modifications among the six $3^{\prime}$ terminal nucleotides of each primer would ensure the detection of viruses from other groups, in particular avian viruses (see Fig. 2B). Modified primers (panINFA-1 and panINFA-23) were predicted to be capable of detecting all Matrix sequences available to date, including strains of avian (including H5N1 viruses) and porcine (including H1N1 viruses) origin, irrespective of hemagglutinin and neuraminidase subtypes.

In conclusion, we propose a simple and cost-effective amplification protocol for the detection of both seasonal influenza virus and newly emerging S-OIV strains. Its effectiveness relies on tried and tested amplification primers that were shown to be extremely reliable for the screening of clinical samples and on actual detection of both seasonal and H1N1 S-OIV in human clinical samples. In silico analysis predicts that minor modifications of these primers would allow the use of this assay for the broad spectrum detection of all currently known variants of influenza A virus, including avian strains.

\section{Disclosure Statement}

No competing financial interests exist.

\section{References}

Brown, IH. Advances in molecular diagnostics for avian influenza. Dev Biol (Basel) 2006; 124:93-97.

Daum, LT, Canas, LC, Arulanandam, BP, Niemeyer, D, et al. Real-time RT-PCR assays for type and subtype detection of influenza A and B viruses. Influenza Other Respi Viruses 2007; 1:167-175.

Gall, A, Hoffmann, B, Harder, T, Grund, C, Beer, M. Universal primer set for amplification and sequencing of HA0 cleavage sites of all influenza A viruses. J Clin Microbiol 2008; 46:25612567.

Gharabaghi, F, Tellier, R, Cheung, R, Collins, C, et al. Comparison of a commercial qualitative real-time RT-PCR kit with direct immunofluorescence assay (DFA) and cell culture for detection of influenza A and B in children. J Clin Virol 2008; 42:190-193.

Karlsson, M, Wallensten, A, Lundkvist, A, Olsen, B, Brytting, M. A real-time PCR assay for the monitoring of influenza A virus in wild birds. J Virol Methods 2007; 144:27-31.

Li, PQ, Zhang, J, Muller, CP, Chen, JX, et al. Development of a multiplex real-time polymerase chain reaction for the detection of influenza virus type A including $\mathrm{H} 5$ and H9 subtypes. Diagn Microbiol Infect Dis 2008; 61:192-197.

Valle, L, Amicizia, D, Bacilieri, S, Banfi, F, et al. Performance testing of two new one-step real time PCR assays for detection of human influenza and avian influenza viruses isolated in humans and respiratory syncytial virus. J Prev Med Hyg 2006; 47:127-133.

van Elden, LJ, Nijhuis, M, Schipper, P, Schuurman, R, van Loon, AM. Simultaneous detection of influenza viruses A and B using real-time quantitative PCR. J Clin Microbiol 2001; 39:196-200.

Whiley, DM, Sloots, TP. A 5'-nuclease real-time reverse transcriptase-polymerase chain reaction assay for the detection of a broad range of influenza A subtypes, including H5N1. Diagn Microbiol Infect Dis 2005; 53:335-337.

Yacoub, A, Kiss, I, Zohari, S, Hakhverdyan, M, et al. The rapid molecular subtyping and pathotyping of avian influenza viruses. J Virol Methods 2009; 156:157-161.

Address correspondence to: Xavier de Lamballerie Unité des Virus Emergents Faculté de Médecine de Marseille Université de la Méditerranée E Institut de Recherche pour le Développement 27 Bd Jean Moulin 13005 Marseille France

E-mail: xavier.de-lamballerie@univmed.fr 